\title{
Influence of branching on the chiral self-assembly of poly(phenylene ethynylene)
}

Joost Steverlynck, Pieter Leysen, Guy Koeckelberghs

Laboratory for Polymer Synthesis, KU Leuven, Celestijnenlaan 200F, B-3001 Heverlee, BELGIUM

Correspondence to: Guy Koeckelberghs (E-mail: guy.koeckelberghs@chem.kuleuven.be)

((Additional Supporting Information may be found in the online version of this article.))

\section{ABSTRACT}

In this contribution we report the synthesis of chiral all-conjugated branched poly(phenylene ethynylenes) with a controlled amount of branching. Subsequently, the self-assembly of these PPEs is studied by means of UV-vis, fluorescence spectroscopy and DSC and the influence of branching is investigated. Finally, CD-spectroscopy is used to study the influence of branching and self-assembly on the chiral expression of these polymers.

KEYWORDS: conjugated polymers, branched, supramolecular structures, self-assembly, chiral

\section{INTRODUCTION}

The properties of conjugated polymers do not only depend on their chemical structure, but to a large extent on their supramolecular organization. Until now mainly linear conjugated polymers have been studied. The reason is twofold. First, the desired optoelectronical properties for the current applications are mostly satisfied by these 1-D systems. Second, the synthesis of these branched systems is synthetically challenging. Nevertheless, branching affects the supramolecular organization of polymers and, as such, can lead to new properties which in turn can make new applications possible. For non-conjugated polymers branching has shown to result in a lower viscosity and globular structure, leading to applications as coating and drug delivery by encapsulation. ${ }^{1}$ For conjugated polymers we could think of solar cell applications. ${ }^{2}$ They are also very promising as active materials for light-emitting devices as they are more stable, have better solubility and do not suffer as much from aggregate-based fluorescence quenching as their linear counterparts. ${ }^{3-11}$ Some even show aggregationinduced emission (AIE). ${ }^{12}$ Crosslinkable hyperbranched polymers are a solution for the solubility problem when preparing organicprocessable multilayer devices. ${ }^{13,14}$ As they are fluorescent and can form a porous network they can be used as highly sensitive chemosensors. ${ }^{15}$ Another interesting property is the possible modification of end-groups allowing tunability of the optical properties. ${ }^{16}$ Although quite some hyperbranched conjugated polymers have been reported ${ }^{17-26}$, no series of all-conjugated branched polymers with a controlled amount of branching has been prepared and a systematic study on the influence of branching on the supramolecular organization is lacking. Very recently the group of Luscombe reported the synthesis of branched P3HT using direct arylation in which the branching can be varied by adjusting the base and ligand. ${ }^{27}$ Finally, hyperbranched PPEPPV copolymers were polymerized by Kub et al. $^{28}$ In this paper, we describe the synthesis of a series of branched PPE's (Figure 1) and 
investigate the influence of branching on the self-assembly and chiral expression.

\section{RESULTS AND DISCUSSION}

\section{Monomer synthesis}

The synthesis of the "linear" monomers $\mathbf{1}$ and $\mathbf{3}$ (Scheme 1) started from hydroquinone which was alkylated twice by the chiral (S)dimethyloctylbromide. ${ }^{29}$ Subsequent iodination renders monomer $\mathbf{1}$. Monomer $\mathbf{3}$ is formed by reaction of monomer $\mathbf{1}$ with trimethylsilylacetylene followed by deprotection of the silyl group with TBAF. $3 \mathrm{H}_{2} \mathrm{O} .{ }^{30}$

The synthesis of the "branched" monomer 4 (scheme 1) started from commercially available 2-iodo-dimethylterephtalate. This product was reduced to the dialcohol with DIBAL and without further purification oxidized with PCC to form 2-iodoterephtaldehyde. ${ }^{31}$ The aldehyde functions were subsequently converted to alkyne functions using the OhiraBestmannreaction.

\section{Polymer synthesis}

The polymers were prepared by means of the Sonogashira reaction. The "linear" $\mathbf{1}$ and $\mathbf{3}$ monomers were combined with the "branched" monomer in well determined quantities (Table 1). lodobenzene (5) was added as a chain stopper. The polymerization was carried out in THF with $\mathrm{Pd}\left(\mathrm{PPh}_{3}\right)_{4}$ as catalyst, $\mathrm{Et}_{3} \mathrm{~N}$ as base and Cul as co-catalyst (Scheme 2). A chain stopper was used to limit the molar mass and crosslinking in order to obtain soluble material and a fixed degree of polymerization of 15 . A similar DP is necessary to compare the different polymers. lodobenzene is used and not phenylacetylene in order to avoid terminal acetylene groups and possible concomitant 2 JOURNAL OF POLYMER SCIENCE PART A: POLYMER CHEMISTRY
Glaser couplings after polymerization. In total, 5 polymers were synthesized which varied in the amount of monomer 4 present in the feed $(0$, $2.5,5,10$ and $20 \%)$, rendering polymers $\mathbf{P 1}, \mathbf{P 2}$, P3, P4 and P5, respectively. After synthesis these polymers are purified by Soxhlet extraction with methanol followed by chloroform. The chloroform fraction was concentrated and the polymer was precipitated in methanol. The yields of the chloroformsoluble fractions are depicted in Table 1. Roughly, the yield drops as the amount of $\mathbf{4}$ in the feed increases, which can be attributed to crosslinking resulting in an insoluble fraction that remained in the Soxhlet thimble. ${ }^{32}$

\section{GPC and NMR}

The dispersities and molar masses (Table 1) were determined via GPC against polystyrene standards and lay in the same range, making a comparison possible. In general, a decrease of molar mass with the degree of branching is observed, which on one hand is in line with a smaller hydrodynamic volume originating from branching, but can on the other hand also be attributed to the fact that only the chloroformsoluble fraction is measured.

Apart from the peak from the aromatic proton of the "linear" unit at 7 ppm $^{33,34}$, also additional peaks are observed, which can be attributed to the branching unit (if present) and terminal units (Figure 2). A complete assignment of the aromatic peaks is shown in Figure 3 for P5 using the ${ }^{1} \mathrm{H}$ NMR spectra of already reported model compounds (Figure S6). Given the fact that iodobenzene is added as chain stopper, two possible end groups are present: either from 5 (signals $\mathrm{g}, \mathrm{h}$, and i) or from monomer $\mathbf{1}$ (signals $e$, and f). Note that no end-groups from 4 are found, which can be explained by its small

(7)WILEY III ONLINE LIBRARY 
presence. The fact that signals e and $f$ diminish with increasing amount of branching is in line with this assignment. The branching unit has resonances at $b, c$ and $d$. The increase of $b$ is clearly in line with increasing amount of $\mathbf{4}$ for P1-> P5. Since each incorporation of $\mathbf{4}$ results in an additional end-group, an increase of the signals $g, h$ and $i$ is expected for P1-> P5. The amount of branching is then calculated from:

$$
\% \text { branching }=\frac{b}{a / 2}
$$

and is tabulated in Table 1. Apart from P5, the amount of branching is nicely reflected in the amount of $\mathbf{4}$ present in the feed. The deviation in case of P5 can be explained by crosslinking and the fact that only the soluble fraction is measured.

The degree of polymerization (DP) can be calculated by dividing the total amount of units present by the number of chains. While the former equals the amount of inner units (either linear or branching units) and the amount of end-groups, the latter can be found from the number of end-groups as follows: a linear chain has two end-groups; therefore, the number of chains equals the amount of end-groups divided by 2 . Since every branching unit increase the number of end-groups by one, the degree of polymerization equals:

$$
D P=\frac{a / 2+b+f+(g+i) / 3}{1 / 2((g+i) / 3+f-b)}
$$

From Table 1., it is clear that all polymers have a similar degree of polymerization (11-12), a little lower than targeted.



WWW.MATERIALSVIEWS.COM

\section{UV-vis}

The self-assembly behavior of P1-P5 was first studied by UV-vis. For each polymer 10 different solutions were made in chloroform adding more and more of the poor solvent methanol. The solvatochromism spectra are found in SI (figures S7-S11). Although no clear redshift was observed, aggregation was visible for $\mathbf{P 1}$ by the appearance of an additional shoulder near $480 \mathrm{~nm}$, which can be attributed to self-assembly and the presence of long-range order in the material (figure 4). ${ }^{35}$ This shoulder is only found for the linear polymer and to a small extent also for P2. However we should note that for $\mathbf{P 2}$ not all chains are branched and this signal is possibly due to the stacking of those non-branched chains. ${ }^{36}$ The other polymers do not show this peak, implying that they do not possess long-range order.

\section{Fluorescence}

A further investigation of the aggregation behaviour was done by fluorescence spectroscopy with excitation at $440 \mathrm{~nm}$. The materials are measured in pure chloroform, in which they are molecularly dissolved, and a mixture of chloroform/methanol (90/10) (figure $3)$. When the spectra in the mixture chloroform and methanol are compared to those in pure chloroform a decrease in intensity of a factor 10 is measured. In addition, an additional peak appears near $\mathbf{4 9 0} \mathbf{n m}$ for $\mathbf{P 1}$, which is not found for the other polymers. This peak has already been attributed in other (linear) PPEs to aggregation. ${ }^{37}$ In the other polymers, this peak is absent or less pronounced. When we study the spectra in mixtures of chloroform/methanol more closely, a systematic decrease in intensity for lower degrees of branching is observed. Clearly, branching shields the polymer chains from each other, reducing the quenching of the 
fluorescence. Hence, these results confirm the results from UV-vis spectroscopy in the sense that branching complicates the aggregation and that even very small amounts of branching disrupts the long-range order.

\section{CD-spectroscopy}

The presence of the chiral alkoxy side-chains allows to investigate the chiral expression. ${ }^{38,39}$ As for UV-vis, for each polymer 10 different solutions were made in chloroform adding more and more methanol (SI, Figures S7-S11). Although aggregation upon addition of a poor solvent was not visible in the UV-vis spectra, the CD-spectra of P1-P5 (figure 4) show clear bisignate $C D$-signals for all polymers. Bisignate Cotton effects result from chiral exciton coupling originating from chirally oriented polymer chains. When the CD-spectra for $\mathbf{P} \mathbf{1}$ are observed more into detail, a bisignate Cotton effect originating from chiral exciton coupling at the $\lambda_{\max }$ of the UV-Vis spectrum with an additional monosignate Cotton-effect, at the wavelength of the shoulder in UV-vis. The latter, pointing at long-range order, is absent or much less pronounced in the other polymers, which is in agreement with the UV-vis spectra. By comparing the intensity of the bisignate Cotton effects in $90 \% \mathrm{MeOH}$ for P1-P5, it is clear that branching in general does not impede chiral expression, but rather increases it. Maximum $\Delta \varepsilon$ values are calculated for $\mathbf{P} \mathbf{3}$ and P5. Altough there is no clear trend between the branching degree and chiral expression, we nevertheless can conclude that chiral expression does not require long-range order. To the contrary, the introduction of branching, leading to the disruption of long-range order, can seemingly cause an increase of the chiral expression of the material. We should note that the chiral signal we analyze does not require 4 JOURNAL OF POLYMER SCIENCE PART A: POLYMER CHEMISTRY long-range order as it is a bisignate Cottoneffect arising from exciton coupling between chiral oriented chains. While perhaps strange at first sight, it has already been found in other systems that molecular imperfections (regioirregularity, achiral units, chiral additive), in chiral materials, can lead to an increase of the chiral expression. ${ }^{40,41,42,43}$

\section{DSC}

The results of the UV-vis and CD-experiments are further confirmed by DSC. While P1 shows clear traces of melting and crystallization (SI, Figure S12), the other polymers did not melt nor crystallize. This is again in agreement with the earlier observations that branching disrupts long-range order.

\section{CONCLUSION}

In conclusion we have developed a synthetic route for the synthesis of an all-conjugated PPE with a controllable degree of branching. These polymers were made by a copolymerization of $A_{2}$ and $B_{2}$ monomers with an $A B_{2}$ monomer via Sonogashira couplings. Polymers with $2.5 \%, 5 \%$, $10 \%$ and $20 \%$ branching monomer were synthesized. Subsequently, the self-assembly of these materials, correlated to their supramolecular structure, was studied by UVvis-, CD-, fluorescence spectroscopy and DSC. It was shown that in all cases self-assembly occurs upon addition of a poor solvent, even for a branching degree up to $20 \%$. On the other hand, the introduction of the slightest amount of branching destroyed the long-range order in the supramolecular structures.

For the chiral expression studied by CDspectroscopy it is found that indeed branching destroys long-range order, but that it does not suppress the chiral expression: the heighest

(WILEY 店 ONLINE LIBRARY 
chiral response is even observed for the most branched material. This result is in agreement with earlier observations that the introduction of a certain amount of defects in a chiral material can improve the chiral expression.

\section{ACKNOWLEDGEMENTS}

We are grateful to the Onderzoeksfonds K.U.Leuven/Research Fund K.U.Leuven and the Fund for Scientific Research (FWO-Vlaanderen) for financial support. JS is grateful to IWT for a doctoral fellowship.

\section{REFERENCES AND NOTES}

(1) Gao, C.; Yan, D. Prog. Polym. Sci. 2004, 29, 183-275.

(2) Qiao, J.; Yang, C.; He, Q.; Bai, F.; Li, Y. J. Appl. Polym. Sci. 2004, 92, 1459-1466.

(3) Xu, M.-H.; Pu, L. Tetrahedron Lett. 2002, 43, 6347-6350.

(4) Lv, M.; Li, S.; Jasieniak, J. J.; Hou, J.; Zhu, J.; Tan, Z.; Watkins, S. E.; Li, Y.; Chen, X. Adv. Mater. Weinheim 2013, 25, 6889-94.

(5) Tang, Y.; Shen, P.; Ding, T.; Huang, H.; Zhao, B.; Tan, S. Eur. Polym. J. 2010, 46, 2033-2041.

(6) Wang, R.; Wang, W.-Z.; Yang, G.-Z.; Liu, T.; Yu, J.; Jiang, Y. J. Polym. Sci., Part A: Polym. Chem. 2008, 46, 790-802.

(7) Taranekar, P.; Qiao, Q.; Jiang, H.; Ghiviriga, I.; Schanze, K. S.; Reynolds, J. R. J. Am. Chem. Soc. 2007, 129, 89589.

(8) Vanjinathan, M.; Lin, H.-C.; Nasar, A. S. J. Polym. Sci., Part A: Polym. Chem. 2012, 50, 3806-3818.
(9) Li, Z.; Ye, S.; Liu, Y.; Yu, G.; Wu, W.; Qin, J.; Li, Z. J Phys Chem B 2010, 114, 9101-8.

(10) Kim, T.; Park, D.-K.; Ahn, T. Mol. Cryst. Liq. Cryst. 2012, 567, 125-131.

(11) Han, Y.; Sun, M.; Fei, Z.; Bo, Z. Chin. Sci. Bull. 2008, 53, 2770-2776.

(12) Chen, J.; Peng, H.; Law, C. C. W.; Dong, Y.; Lam, J. W. Y.; Williams, I. D.; Tang, B. Z. Macromolecules 2003, 36, 43194327.

(13) Paul, G. K.; Mwaura, J.; Argun, A. a.; Taranekar, P.; Reynolds, J. R. Macromolecules 2006, 39, 7789-7792.

(14) Tang, D.-F.; Wen, G.-A.; Qi, X.-Y.; Wang, H.-Y.; Peng, B.; Wei, W.; Huang, W. Polymer 2007, 48, 4412-4418.

(15) Wu, X.; Li, H.; Xu, B.; Tong, H.; Wang, L. Polymer Chemistry 2014.

(16) Song, L.; Tu, C.; Shi, Y.; Qiu, F.; He, L.; Jiang, Y.; Zhu, Q.; Zhu, B.; Yan, D.; Zhu, X. Macromol. Rapid Commun. 2010, 31, 443-8.

(17) Richter, T. V.; Bühler, C.; Ludwigs, S. J. Am. Chem. Soc. 2012, 134, 43-6.

(18) Qu, J.; Shiotsuki, M.; Kobayashi, N.; Sanda, F.; Masuda, T. Polymer 2007, 48, 6481-6490.

(19) Grigoras, M.; Stafie, L. High Perf. Polym. 2008, 21, 304-314.

(20) Lin, T.; He, Q.; Bai, F.; Dai, L. Thin Solid Films 2000, 363, 122-125.

Kim, Y. H.; Webster, O. W. J. Am. Chem. Soc. 1990, 112, 4592-4593.



WWW.MATERIALSVIEWS.COM 
(22) Kim, Y. H.; Webster, O. W. Macromolecules 1992, 25, 5561-5572.

(23) Law, C. C. W.; Chen, J.; Lam, J. W. Y.; Peng, H.; Tang, B. Z. J. Inorg. Organomet. Polym. 2004, 14, 39-51.

(24) He, Q.; Huang, H.; Yang, J.; Lin, H.; Bai, F. J. Mater. Chem. 2003, 13, 1085-1089.

(25) Yao, J.; Son, D. Y. Organometallics 1999, 18, 1736-1740.

(26) Peng, H.; Lam, J. W. Y.; Tang, B. Z. Macromol. Rapid Commun. 2005, 26, 673-677.

(27) Okamoto, K.; Housekeeper, J. B.; Michael, F. E.; Luscombe, C. K. Polymer Chemistry 2013, 4, 3499.

(28) Kub, C.; Tolosa, J.; Zucchero, A. J.; McGrier, P. L.; Subramani, C.; Khorasani, A.; Rotello, V. M.; Bunz, U. H. F. Macromolecules 2010, 43, 21242129.

(29) Bidan, G.; Guillerez, S.; Sorokin, V. Adv. Mater. 1996, 8, 157-160.

(30) Ajayaghosh, A.; Varghese, R.; Mahesh, S.; Praveen, V. K. Angew. Chem. Int. Ed. 2006, 45, 7729-32.

(31) Wu, Y.; Guo, H.; Zhang, X.; James, T. D.; Zhao, J. Chemistry 2011, 17, 763244.

(32) Hittinger, E.; Kokil, A.; Weder, C. Angew. Chem. Int. Ed. 2004, 43, 180811.

(33) Francke, V.; Räder, H. J.; Geerts, Y.; Müllen, K. Macromol. Rapid Commun. 1998, 19, 275-281.
(34) Li, H.; Powell, D. R.; Hayashi, R. K.; West, R. Macromolecules 1998, 31, 5258.

(35) Zahn, S.; Swager, T. M. Angew. Chem. Int. Ed. 2002, 41, 4225-30.

$2,5 \%$ of 4 , while degree of polymerization is only 15 .

(37) Halkyard, C. E.; Rampey, M. E.; Kloppenburg, L.; Studer-Martinez, S. L.; Bunz, U. H. F. Macromolecules 1998, 31, 8655-8659.

(38) Fiesel, R.; Scherf, U. Macromol. Rapid Commun. 1998, 19, 427-431.

(39) Fiesel, R.; Scherf, U. Acta. Polym. 1998, 49, 445-449.

(40) Verswyvel, M.; Monnaie, F.; Koeckelberghs, G. Macromolecules 2011, 44, 9489-9498.

(41) Willot, P.; Steverlynck, J.; Moerman, D.; Leclère, P.; Lazzaroni, R.;

Koeckelberghs, G. Polymer Chemistry 2013, 4, 2662.

(42) Watanabe, K.; Koyama, Y.; Suzuki, N.; Fujiki, M.; Nakano, T. Polymer Chemistry 2014, 5, 712 .

Liu, J.; Zhang, J.; Zhang, S.; Suzuki, N.; Fujiki, M.; Wang, L.; Li, L.; Zhang, W.; Zhou, N.; Zhu, X. Polymer Chemistry 2014, 5, 784 . 


\section{Captions to figures}

FIGURE 1: Structure of the polymers

FIGURE 2: ${ }^{1} \mathrm{H}-\mathrm{NMR}$ spectra of P1-P5 for $\mathrm{A}$ ) aromatic and $\mathrm{B}$ ) aliphatic region in $\mathrm{CDCl}_{3}$.

FIGURE 3: Assignment of the aromatic region of the ${ }^{1} \mathrm{H}$ NMR spectrum of P5.

FIGURE 4: (A) UV-vis spectra of P1-P5 (B) fluorescence spectra and (C) $\mathrm{CD}$ spectra in $100 \% \mathrm{CHCl}_{3}$ and $90 \%$ in $\mathrm{MeOH} / 10 \%$ in $\mathrm{CHCl}_{3}$, the fluorescene spectra are corrected for absorption.

\section{Captions to schemes}

SCHEME 1: Synthesis of the monomers

SCHEME 2: Synthesis of the polymers

\section{Captions to tables}

TABLE 1: Ratio of monomers used, yields, number-averaged molar mass, dispersity, degree of branching and degree of polymerization 


\section{GRAPHICAL ABSTRACT}

Joost Steverlynck, Pieter Leysen, Guy Koeckelberghs

Influence of branching on the chiral self-assembly of poly(phenylene ethynylene)

A series of all-conjugated poly(phenylene ethynylenes) with a controllable degree of branching is prepared. Subsequently, by using UV-vis, CD, fluorescence spectroscopy and DSC the influence of branching on the self-assembly is studied. Finally, it is investigated how branching and the resulting change in self-assembly affects the chiral expression.

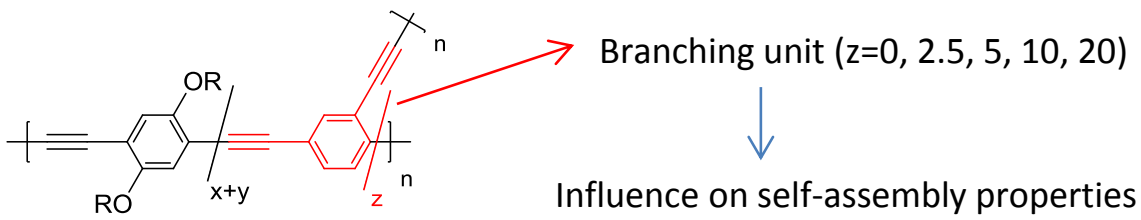

